\title{
Measurement of the energy spectrum of cosmic-ray helium with CALET on the International Space Station
}

\author{
Paolo Brogi ${ }^{a, b, *}$ and K. Kobayashi ${ }^{c, d}$ on behalf of the CALET Collaboration \\ (a complete list of authors can be found at the end of the proceedings) \\ a INFN Sezione di Pisa, Polo Fibonacci, Largo B. Pontecorvo, 3, 56127 Pisa, Italy \\ ${ }^{b}$ Department of Physical Sciences, Earth and Environment, University of Siena, via Roma 56, 53100 \\ Siena, Italy \\ ${ }^{c}$ Waseda Research Institute for Science and Engineering, Waseda University, 17 Kikuicho, Shinjuku, Tokyo \\ 162-0044, Japan \\ dJEM Utilization Center, Human Spaceflight Technology Directorate, Japan Aerospace Exploration \\ Agency, 2-1-1 Sengen, Tsukuba, Ibaraki 305-8505, Japan \\ E-mail: paolo.brogi@unisi.it
}

The CALorimetric Electron Telescope (CALET) is a space instrument designed to carry out precision measurements of high energy cosmic-rays. It was installed onboard the International Space Station in August 2015 and since mid-October 2015 it is collecting data with excellent performance and no significant interruptions. The instrument consists of two layers of segmented plastic scintillators to identify the charge of individual elements from proton to iron, followed by a thick (30 $X_{0}$ and $\sim 1.3 \lambda_{I}$ ) calorimeter. It comprises a finely segmented imaging calorimeter $\left(3 X_{0}\right)$, providing accurate particle tracking and complementary charge measurement, and a total absorption $\left(27 X_{0}\right)$ homogeneous calorimeter. In addition to its primary science goal of identifying nearby sources of high-energy electrons and possible signatures of dark matter in the electron spectrum, CALET is carrying out measurements of the energy spectra, relative abundances and secondary-to-primary ratios of individual elements from proton to iron and above, in order to shed light on the mechanism of acceleration and propagation of cosmic rays in the Galaxy. Preliminary measurements of the energy spectrum of cosmic-ray helium, based on the first five years of collected data, will be presented and details of the analysis are given. The observations performed by CALET in the energy interval from a few $\mathrm{GeV} / \mathrm{n}$ to the multi-TeV region show that the helium differential spectrum does not follow a simple power-law.

$37^{\text {th }}$ International Cosmic Ray Conference (ICRC 2021)

July 12 th - 23rd, 2021

Online - Berlin, Germany

\footnotetext{
${ }^{*}$ Presenter
} 


\section{Introduction}

The CALorimetric Electron Telescope (CALET) $[1,2]$ is a space-based experiment developed and operated by an international collaboration led by the Japanese Space Agency (JAXA) with the participation of the Italian Space Agency (ASI) and NASA. The CALET detector was installed on the Japanese Experiment Module Exposure Facility (JEM-EF) onboard the International Space Station (ISS) since August 2015, and after a preliminary commissioning phase, it is taking data smoothly form October 2015.

The CALET science program is wide and addresses several outstanding questions of high-energy astroparticle physics including the origin of cosmic rays (CR); the possible presence of nearby astrophysical CR sources; the study of their acceleration mechanism(s); the propagation of primary and secondary elements in the galaxy; the nature of dark matter and its localization. Moreover, the CALET telescope is also capable to detect gamma-ray transients trough a dedicated instrument (CGBM) that covers the energy range $7 \mathrm{keV}-20 \mathrm{MeV}$, and could be used, in combination with the calorimeter, to perform the search of counterpart emission related to gravitational wave events [3, 4]. Taking advantage of its wide dynamic range, large thickness and excellent charge identification capability, CALET is carrying out extensive measurements of individual chemical elements in CR up to iron, in the energy range from few $\mathrm{GeV}$ up to the $\mathrm{PeV}$ region.

In this paper, we describe the analysis procedure for the Helium flux measurement, and we present the preliminary results based on the data collected in 1815 days of CALET operation onboard the ISS.

\section{The CALET telescope}

The CALET main telescope, is an all-calorimetric instrument, that consists of three subdetectors. The CHarge Detector (CHD), that is positioned at the top of the apparatus and consists of a two layer hodoscope of plastic scintillators paddles (14 paddles for each layer): this first subdetector performs the charge identification of individual nuclear species, providing a measurement of the charge $Z$ of the incident particle over a wide dynamic range (from $Z=1$ up to $Z=40$ ) [5]. The IMaging Calorimeter (IMC), a fine grained sampling calorimeter segmented longitudinally into 16 layers of scintillating fibers (with $1 \mathrm{~mm}^{2}$ square cross-section) read out individually and arranged in pairs along orthogonal directions, each pair is interleaved with thin tungsten absorbers (for a total thickness of $3 X_{0}$ ). It is used to reconstruct the early shower profile and the impinging particle trajectory with good angular resolution and a redundant charge measurement [6]. The third detector is the Total AbSorption Calorimeter (TASC), an homogeneous calorimeter made of 12 layers of lead-tungstate (PWO) logs, arranged in pairs along $x-y$ directions, and capable, with its $27 X_{0}$ thickness and its shower imaging capability, to measure electrons and gamma-rays with an excellent energy resolution, providing high discrimination against hadronic cascades.

The total thickness of the main telescope is equivalent to $30 X_{0}$ and 1.3 proton interaction lengths $\left(\lambda_{I}\right)$, the geometrical factor is $0.12 \mathrm{~m}^{2}$ sr. A more detailed description of the instrument can be found in [7] and in the Supplemental Material (SM) of [8]. 

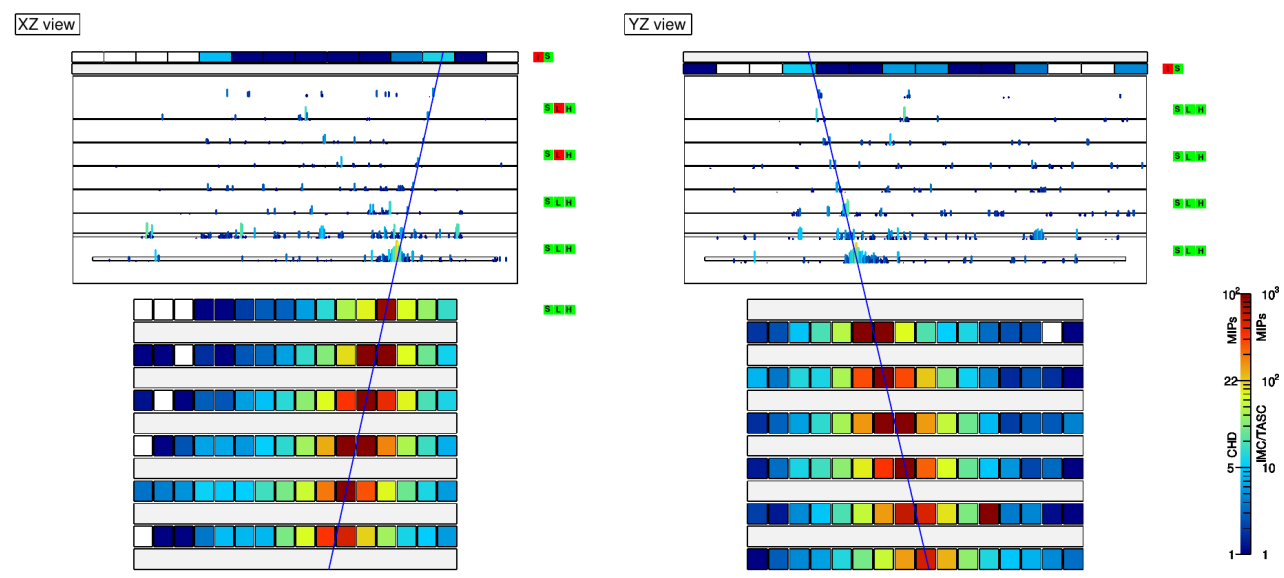

Figure 1: CALET event display of a selected helium candidate, with a shower energy in TASC of about 700 $\mathrm{GeV}$, crossing the tree sub-detectors. The blue lines represent the projections of the reconstructed impinging particle trajectory in the $X-Z$ and $Y-Z$ views respectively.

\section{Data analysis}

In the analysis reported here, we use 1815 days of flight data (FD) collected from October 13, 2015 to September 30, 2020.

The raw signal of each detector channel is carefully calibrated using penetrating protons and $\mathrm{He}$ particles, selected in-flight by a dedicated trigger mode in order to correct for non-uniformity in light output, gain differences among the channels, position, temperature and temporal gain variations [7]. For each CR event the impinging particle track, charge and energy are then reconstructed. This allows to select the helium sample, sorted into energy intervals, in order to compute the energy spectrum. In figure 1 it is represented an event display of a selected helium candidate crossing the three CALET sub-detectors. The blue lines represent the projections of the reconstructed particle trajectory in the $X-Z$ and $Y-Z$ instrument views, respectively.

Two detailed Monte Carlo (MC) simulations, based on EPICS [9] and FLUKA [10] packages were developed, they are used to validate and tune the reconstruction method and evaluate event reconstruction efficiencies, background contaminations and the energy response matrix. The main steps of the analysis procedure are described in the following subsections.

\subsection{Preselection}

As first step, well reconstructed and well contained events were selected on the basis of the following criteria: Trigger: in this analysis, we have used events taken with the on-board high-energy (HE) trigger mode, designed to ensure maximum exposure to electrons above $10 \mathrm{GeV}$ and other high-energy shower events. Since the HE trigger can be affected by position and temperature dependence, as well as temporal gain variations, an offline trigger confirmation is applied, requiring sufficiently severer conditions than the HE trigger. Track quality cut: tracks are reconstructed using a combinatorial Kalman Filter (KF) algorithm [11]. This method exploits the fine granularity and imaging capability of the IMC and provides robust track finding and fitting, allowing to identify 
the incident CR track in a large amount of shower particle tracks back-scattered from TASC. The algorithm has been validated with simulated data and good agreement was found between MC and FD distributions of reconstructed incident angle and Impact Point (IP) coordinates on top of CHD. For $\mathrm{He}$ nuclei we found an angular resolution of about $0.1^{\circ}$ and a resolution on the IP on CHD of $\sim 400 \mu \mathrm{m}$. Geometrical condition: The reconstructed events are required to pass through the whole detector, i.e., from CHD top to TASC bottom, with $2 \mathrm{~cm}$ clearance from the edges of the TASC top layer. Within a fiducial region, known as acceptance A1, limited to a Geometric Factor of $0.051 \mathrm{~m}^{2} \mathrm{sr}$ ( $49 \%$ of the total GF). Electron rejection: Electron contamination in the helium sample after the charge selection is not an issue. Anyway, since the proton sample is used to correct charge contamination (that is relevant), we adopt an electron rejection cut. The cut is based on the energy deposits found inside one Moliere radius around each IMC fiber matched to the track and on the energy deposit in the last TASC layer. Most of the electrons are rejected while retaining a very high efficiency for helium nuclei. Off-Acceptance Rejection (OAR) cuts: A number of events are erroneously reconstructed inside the fiducial acceptance A1, while the true acceptance is different. To reject most of these events, different cuts are applied. The fractional energy deposit in each one of the first two TASC layers is required to be less than 0.3, to reject laterally incident tracks. A consistency within a PWO $\log$ is required between the impact points of a track onto the first two layers of the TASC and the center of gravity of the corresponding energy deposits. Exploiting the TASC granularity, the shower axis is reconstructed with the method of moments (see [12] for details), and is required to cross the TASC-X1 layer. This reject, with very high efficiency, lateral events erroneously reconstructed inside the fiducial region.

\subsection{Charge identification}

In CALET, the charge is measured with two independent subsystems that are routinely used to cross-calibrate each other: the CHD and the IMC. The tracking information is used to select the CHD paddles crossed by the primary particle, after application of position and time-dependent calibrations and corrections [7] the information from the two CHD layers is combined into a single charge estimator. The IMC, being equipped with individually readout scintillating fibers, has a suitable granularity not only to provide excellent tracking capabilities, but also to sample the ionization deposits along the track in each layer, providing a multiple $d E / d x$ measurement with a maximum of 16 samples. The interaction point (IP) is first reconstructed [6] and only the $d E / d x$ ionization clusters from the layers upstream the IP are used. The charge is evaluated as the truncated-mean of the valid samples rejecting the $30 \%$ of the higher ones. The non-linear response due to the saturation of the scintillation light in the fibers is corrected, for both IMC and CHD, by fitting the light yield according to the halo model of $[13,14]$. Moreover, both charge measurements are calibrated for the energy shift (related to the energy increasing back-splash background), so that the peak position of charge distributions does not depend from the shower energy deposit in the TASC. To have a perfect agreement between FD and MC, the MC data are fine tuned over FD, fitting proton and helium charge distributions in several energy slices with an asymmetric "Lan-gauss" distribution (Landau* Gaussian), see the left panel of figure 2 for an example. The FWHM and peak position of the charge distribution are extracted for each energy slice, together with the Left and Right handed half Width at Half Maximum (LWHM, RWHM), and fitted to the whole energy range with a logarithmic polynomial (right panel of figure 2). The fits to peak position and FWHM 

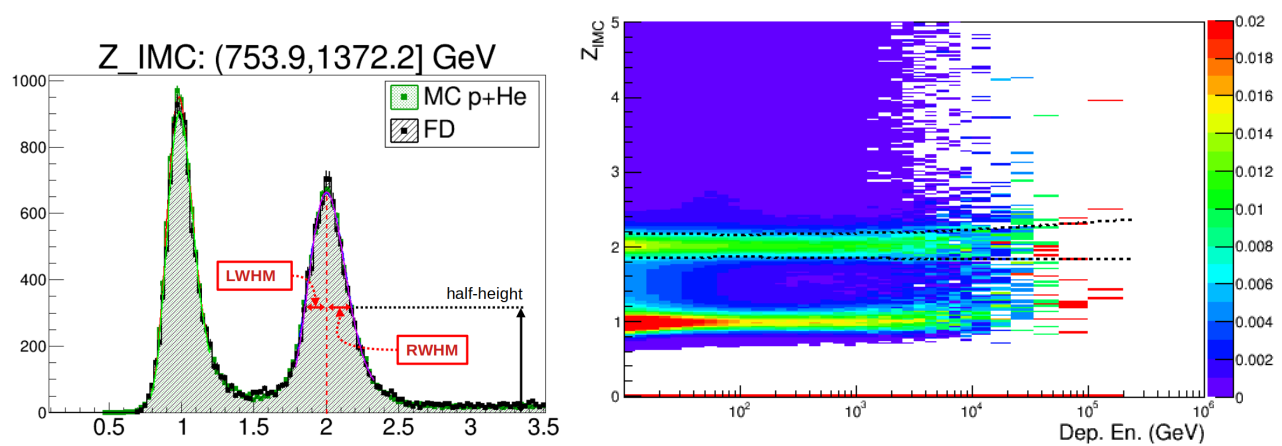

Figure 2: Left panel: the IMC charge distribution in a given energy slice for FD (black) and the sum of proton and helium MC (green). "Lan-gauss" fit to the charge peak distribution is represented as example, were the left and right width at half maximum of the curve are highlighted. Right panel: the IMC charge distribution dependence from the TASC shower energy deposit is shown, the black dashed line shows the width of the distribution.

values are used, on an event by event basis, to fine tune the MC distributions. The fits to LWHM and RWHM values are used to perform an energy dependent charge cut that allows to obtain an almost flat charge selection efficiency (roughly 65\%). For this analysis, the charge selection of helium candidates is performed by applying simultaneous window cuts on CHD and IMC reconstructed charges, requiring $3 \cdot \mathrm{LWHM}<Z_{C H D}<5 \cdot$ RWHM and $3 \cdot \mathrm{LWHM}<Z_{I M C}<5 \cdot$ RWHM.

\subsection{Background subtraction}

Background contamination is estimated from the MC simulation of protons, helium and from FD, as a function of the observed energy. Available spectral data, e.g. from [15-17], are used to simulate their spectral shape. The MC simulations are used to evaluate the relative contributions, and the FD to assess the proton and helium relative abundances. The dominant component is the charge contamination from protons misidentified as helium. Other not negligible contributions are from off-acceptance helium and protons mis-reconstructed inside acceptance A1. The estimated background is then subtracted bin by bin from the measured helium candidate distribution $(\mathrm{dN} / \mathrm{dE})$, prior to the unfolding procedure used to reconstruct the primary particle energy starting from the measured shower energy in TASC.

\subsection{Energy measurement}

The shower energy of each event is calculated as the sum of the calibrated energy deposits of all the TASC channels. Unlike for electrons, the energy released in TASC by interacting CR nuclei is only a fraction of the primary particle energy with large event-to-event fluctuations. For flux measurement, energy unfolding is applied to correct $\mathrm{dN} / \mathrm{dE}$ distribution of selected helium candidates for significant bin-to-bin migration effects (due to the limited energy resolution) and infer the primary particle energy. In this analysis, we apply the iterative unfolding method based on the Bayes theorem [18] implemented in the RooUnfold package [19]. The response matrix represents the probability that primary nuclei in a certain energy interval of the CR spectrum produce an energy deposit in a given bin of deposited energy. This "smearing" matrix is derived 
using the MC simulation after applying the same selection criteria as for FD, and is used in the unfolding procedure. The simulated energy response was validated and tuned with beam test data, taken as CERN SPS in 2015 using a beam of accelerated ion fragments with $A / Z=2$ and kinetic energy $150 \mathrm{GeV} / \mathrm{n}$. The energy bin width is chosen commensurate with the RMS resolution of TASC ( $30 \%$ for nuclei).

\subsection{Preliminary systematic evaluation}

The dominant sources of systematic uncertainties in helium analysis can be grouped into energy independent and energy dependent contributions. The former include systematic effects in normalization that were studied in details here [8] and is estimated as $4.1 \%$. The latter includes contributions from: Trigger: the absolute calibration of the trigger efficiency was performed at the beam test. The main source of uncertainty comes from the accuracy of the calibration. Possible systematic bias due to normalization in the measurements of trigger efficiency was considered as uncertainty. Shower energy correction: as in the case of the uncertainty on the trigger efficiency, the absolute calibration of the energy response was performed using the beam test data in the low-energy region. Both the contribution coming from the accuracy of the calibration and from the model used to fit the test beam data are taken into account to assess this systematic. Track reconstruction and acceptance: tracking can affect many aspects of the analysis, the effects on the flux are evaluated by studying its dependence on pre-selection cuts relative to goodness-of-tracking. To investigate the uncertainty on the acceptance, restricted acceptance regions have been studied and the resultant fluxes compared. Background subtraction: background subtraction is found to be slightly dependent from the simulated spectral shape, several re-weighting function were adopted to vary the MC spectrum, the relative differences with respect to the reference case were considered as systematic uncertainty for each energy bin. Unfolding: possible source of systematic error associated to the energy unfolding are: the adopted deconvolution algorithm, the parameter used in the chosen algorithm and the assumed MC spectrum used to build the smearing matrix. All these possible source of error are investigated separately, and then summed (in quadrature) to obtain the systematic error. Charge ID and Off-Acceptance Rejection cuts: it is very important to study the flux stability against selection cuts efficiencies. The thresholds of each cut has been varied separately in a reasonable rage around the reference value, and the differences between the reference case and all the other cases were considered as systematic uncertainty. MC model: in this analysis we perform a preliminary evaluation of the systematic uncertainty related to the MC model using a second Monte Carlo (i.e. Fluka) to compare with the adopted one (Epics). For each bin, this systematic error is obtained using Fluka to evaluate: the shower energy correction, the smearing matrix, and some relevant selection efficiencies.

Two independent helium analyses were carried out by separate groups inside the CALET collaboration, using different event selection and background rejection procedure, preliminary results are consistent within the errors.

\section{Preliminary helium energy spectrum}

The helium energy spectrum is calculated as: $\Phi(\tilde{E})=\frac{N(E)}{\Delta E \times \varepsilon(E) \times \Omega \times L T}$ where $\tilde{E}$ is the median kinetic energy of the $[E, E+\Delta E$ ) bin, $\Delta E$ is the energy bin width, $\varepsilon(E)$ the overall selection 


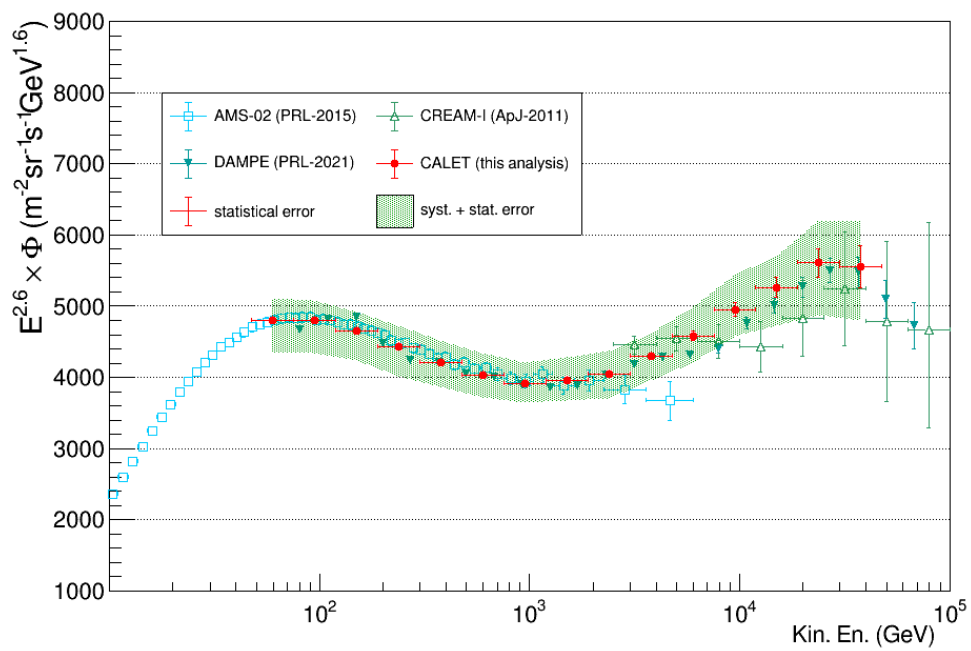

Figure 3: Preliminary helium flux measurement with CALET (red markers), compared with previous direct observations $[16,17,20]$. The error bars represent only statistical error, the green band represents statistical and systematic error.
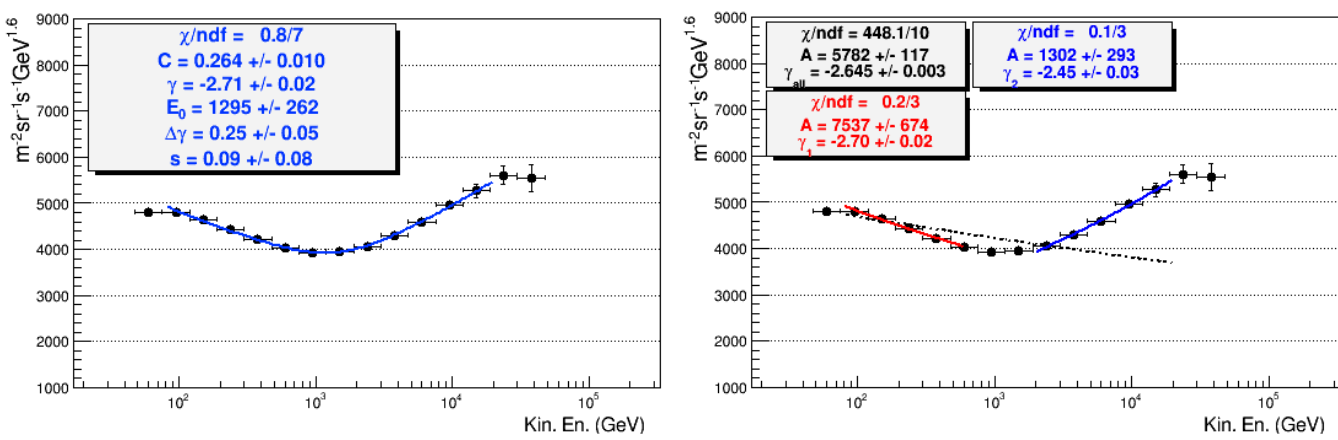

Figure 4: Very preliminary fit of CALET data, with only statistical error included. Left panel: a "smoothly broken power-law" [17] function is used, the result is consistent with DAMPE [20] within the errors. Right panel: a single power low function is used in two sub-ranges of $[80,600] \mathrm{GeV},[2,20] \mathrm{TeV}$ and in the full range. The sub-ranges are individually compatible with a single power law function, the whole range is not.

efficiency, and $L T$ is the live time ( $\sim 85 \%$ of total observation time), $\Omega$ the "fiducial" geometrical acceptance $\left(\sim 510 \mathrm{~cm}^{2} \mathrm{sr}\right), N(E)$ the bin content in the unfolded distribution.

The preliminary energy spectrum of helium nuclei in CR, as measured with CALET in 1815 days of operation is shown in figure 3 covering an interval of kinetic energy per particle from $\sim 50 \mathrm{GeV}$ to $\sim 50 \mathrm{TeV}$, compared with previous observations from space-based [17, 20] and balloon-borne [16] experiments. A very preliminary fit of CALET data, with only statistical error included, has been performed in the energy range from $80 \mathrm{GeV}$ to $20 \mathrm{TeV}$. The sub-ranges of $[80,600] \mathrm{GeV}$ and $[2,20] \mathrm{TeV}$ can be fitted individually with a single power law function, but not the whole range. A progressive hardening up to the multi-TeV region was observed, and the fit with a "smoothly broken power-law" function [17] gives a power law index $(\gamma), \Delta \gamma$ and break energy $\left(E_{0}\right)$ consistent, within 
the errors, with the most recent results of DAMPE [20]. These preliminary results are reported in the left and right panels of figure 4 respectively.

\section{Conclusions}

The preliminary measurement of the helium spectrum demonstrates the excellent capability of CALET to resolve spectral features in the CR spectra from few tens of $\mathrm{GeV}$ up to tens of $\mathrm{TeV}$.

Further analysis with an increased data set and including a detailed assessment of systematic uncertainties, is ongoing, aimed at investigating possible spectral features above $20 \mathrm{TeV}$. An additional study is ongoing to assess the effect of the $\mathrm{He}^{3}$ contribution to the total helium flux.

\section{References}

[1] Y. Asaoka et al. (CALET Collab.), J. Phys.: Conf. Ser. 1181, 012003 (2019).

[2] P. Brogi et al. (CALET Collab.) Phys. Scr. 95, 074012 (2020).

[3] O. Adriani et al. (CALET Collab.), Astrophys. J. Lett. 863, 160 (2018).

[4] O. Adriani et al. (CALET Collab.), Astrophys. J. Lett. 829, L20 (2016).

[5] P. S. Marrocchesi et al., Nucl. Instrum. Methods A 659, 477 (2011).

[6] P. Brogi et al. , PoS (ICRC2015) 595 (2015)

[7] Y. Asaoka et al. (CALET Collab.), Astropart. Phys. 91, 1 (2017).

[8] O. Adriani et al. (CALET Collab.), Phys. Rev. Lett. 119, 181101 (2017).

[9] K. Kasahara, Proc. of 24 th ICRC, Vol. 1 (1995) 399

[10] T. T. Böhlen et al., Nuclear Data Sheets 120 (2014) 211

[11] P. Maestro and N. Mori (for the CALET Collab.), PoS (ICRC2017) 208 (2017).

[12] J. J. Gomez et al., Nucl. Instr. Meth. Phys. Res. A, 262(2-3), 284-290, 1987.

[13] R. Voltz, J. Lopes da Silva, G. Laustriat and A. Coche, JChPh 45 (1966) 3306

[14] G. Tarle et al., Nuclear Instruments and Methods in Physics Research B6 504-512, 1985

[15] M. Aguilar et al. (AMS Collab.), Phys. Rev. Lett. 114, 171103 (2015).

[16] Y. S. Yoon et al., Astrophys. J. 728, 122 (2011); Astrophys. J. 839, 5 (2017).

[17] M. Aguilar et al. (AMS Collab.), Phys. Rev. Lett. 115, 211101 (2015).

[18] G. D’Agostini, Nucl. Instr. and Meth. A 362 (1995) 487

[19] T. Adye, arXiv:1105.1160v1 (2011)

[20] F. Alemanno et al. (DAMPE Collab.), Phys. Rev. Lett. 126, 201102 (2021). 


\section{Full Authors List: CALET Collaboration}

O. Adriani ${ }^{1,2}$, Y. Akaike ${ }^{3,4}$, K. Asano ${ }^{5}$, Y. Asaoka ${ }^{5}$, E. Berti ${ }^{1,2}$, G. Bigongiari ${ }^{6,7}$, W. R. Binns ${ }^{8}$, M. Bongi ${ }^{1,2}$, P. Brogi ${ }^{6,7}$, A. Bruno ${ }^{9,10}$, J. H. Buckley ${ }^{8}$, N. Cannady ${ }^{11,12,13}$, G. Castellini ${ }^{14}$, C. Checchia ${ }^{6}$, M. L. Cherry ${ }^{15}$ G. Collazuol ${ }^{16,17}$, K. Ebisawa ${ }^{18}$, A. W. Ficklin ${ }^{15}$, H. Fuke ${ }^{18}$, S. Gonzi ${ }^{12}{ }^{2}$, T. G. Guzik ${ }^{15}$, T. Hams ${ }^{11}$, K. Hibino ${ }^{19}$, M. Ichimura ${ }^{20}$, K. Ioka $^{21}$, W. Ishizaki ${ }^{5}$, M. H. Israel ${ }^{8}$, K. Kasahara ${ }^{22}$, J. Kataoka ${ }^{23}$, R. Kataoka ${ }^{24}$, Y. Katayose ${ }^{25}$, C. Kato $^{26}$, N. Kawanaka ${ }^{27,28}$, Y. Kawakubo ${ }^{15}$, K. Kobayashi ${ }^{3,4}$, K. Kohri ${ }^{29}$, H. S. Krawczynski ${ }^{8}$, J. F. Krizmanic ${ }^{11,12,13}$, P. Maestro, 6, P. S. Marrocchesi 6,7 , A. M. Messineo ${ }^{30,7}$, J.W. Mitchell ${ }^{12}$, S. Miyake ${ }^{32}$, A. A. Moiseev ${ }^{33,12,13}$ M. Mori ${ }^{34}$, N. Mori ${ }^{2}$, H. M. Motz ${ }^{35}$, K. Munakata ${ }^{26}$, S. Nakahira ${ }^{18}$, J. Nishimura ${ }^{18}$, G. A. de Nolfo ${ }^{9}$, S. Okuno ${ }^{19}$, J. F. Ormes $^{36}$, N. Ospina ${ }^{16,17}$, S. Ozawa ${ }^{37}$, L. Pacini ${ }^{1,14,2}$, P. Papini ${ }^{2}$, B. F. Rauch ${ }^{8}$, S. B. Ricciarini ${ }^{14,2}$, K. Sakai ${ }^{11,12,13 \text {, }}$ T. Sakamoto ${ }^{38}$, M. Sasaki ${ }^{33,12,13}$, Y. Shimizu ${ }^{19}$, A. Shiomi ${ }^{39}$, P. Spillantini ${ }^{1}$, F. Stolzi ${ }^{6,7}$, S. Sugita ${ }^{38}$, A. Sulaj ${ }^{6,7}$, M. Takita ${ }^{5}$, T. Tamura ${ }^{19}$, T. Terasawa ${ }^{40}$, S. Torii ${ }^{3}$, Y. Tsunesada ${ }^{41}$, Y. Uchihori ${ }^{42}$, E. Vannuccini ${ }^{2}$, J. P. Wefel ${ }^{15}$, K. Yamaoka ${ }^{43}$, S. Yanagita ${ }^{44}$, A. Yoshida ${ }^{38}$, K. Yoshida ${ }^{22}$, and W. V. Zober ${ }^{8}$

${ }^{1}$ Department of Physics, University of Florence, Via Sansone, 1, 50019 Sesto, Fiorentino, Italy, ${ }^{2}$ INFN Sezione di Florence, Via Sansone, 1, 50019 Sesto, Fiorentino, Italy, ${ }^{3}$ Waseda Research Institute for Science and Engineering, Waseda University, 17 Kikuicho, Shinjuku, Tokyo 162-0044, Japan, ${ }^{4}$ JEM Utilization Center, Human Spaceflight Technology Directorate, Japan Aerospace Exploration Agency, 2-1-1 Sengen, Tsukuba, Ibaraki 305-8505, Japan, ${ }^{5}$ Institute for Cosmic Ray Research, The University of Tokyo, 5-1-5 Kashiwa-no-Ha, Kashiwa, Chiba 277-8582, Japan, ${ }^{6}$ Department of Physical Sciences, Earth and Environment, University of Siena, via Roma 56, 53100 Siena, Italy, ${ }^{7}$ INFN Sezione di Pisa, Polo Fibonacci, Largo B. Pontecorvo, 3, 56127 Pisa, Italy, ${ }^{8}$ Department of Physics and McDonnell Center for the Space Sciences, Washington University, One Brookings Drive, St. Louis, Missouri 63130-4899, USA, ${ }^{9}$ Heliospheric Physics Laboratory, NASA/GSFC, Greenbelt, Maryland 20771, USA, ${ }^{10}$ Department of Physics, Catholic University of America, Washington, DC 20064, USA, ${ }^{11}$ Center for Space Sciences and Technology, University of Maryland, Baltimore County, 1000 Hilltop Circle, Baltimore, Maryland 21250, USA, ${ }^{12}$ Astroparticle Physics Laboratory, NASA/GSFC, Greenbelt, Maryland 20771, USA, ${ }^{13}$ Center for Research and Exploration in Space Sciences and Technology, NASA/GSFC, Greenbelt, Maryland 20771, USA, ${ }^{14}$ Institute of Applied Physics (IFAC), National Research Council (CNR), Via Madonna del Piano, 10, 50019 Sesto, Fiorentino, Italy, ${ }^{15}$ Department of Physics and Astronomy, Louisiana State University, 202 Nicholson Hall, Baton Rouge, Louisiana 70803, USA, ${ }^{16}$ Department of Physics and Astronomy, University of Padova, Via Marzolo, 8, 35131 Padova, Italy, ${ }^{17}$ INFN Sezione di Padova, Via Marzolo, 8, 35131 Padova, Italy, ${ }^{18}$ Institute of Space and Astronautical Science, Japan Aerospace Exploration Agency, 3-1-1 Yoshinodai, Chuo, Sagamihara, Kanagawa 252-5210, Japan, ${ }^{19}$ Kanagawa University, 3-27-1 Rokkakubashi, Kanagawa, Yokohama, Kanagawa 221-8686, Japan, ${ }^{20}$ Faculty of Science and Technology, Graduate School of Science and Technology,, Hirosaki University, 3, Bunkyo, Hirosaki, Aomori 036-8561, Japan, ${ }^{21}$ Yukawa Institute for Theoretical Physics, Kyoto University, Kitashirakawa Oiwakecho, Sakyo, Kyoto 606-8502, Japan, ${ }^{22}$ Department of Electronic Information Systems, Shibaura Institute of Technology, 307 Fukasaku, Minuma, Saitama 337-8570, Japan, ${ }^{23}$ School of Advanced Science and Engineering, Waseda University, 3-4-1 Okubo, Shinjuku, Tokyo 169-8555, Japan, ${ }^{24}$ National Institute of Polar Research, 10-3, Midori-cho, Tachikawa, Tokyo 190-8518, Japan, ${ }^{25}$ Faculty of Engineering, Division of Intelligent Systems Engineering, Yokohama National University, 79-5 Tokiwadai, Hodogaya, Yokohama 240-8501, Japan, ${ }^{26}$ Faculty of Science, Shinshu University, 3-1-1 Asahi, Matsumoto, Nagano 390-8621, Japan, ${ }^{27}$ Hakubi Center, Kyoto University, Yoshida Honmachi, Sakyo-ku, Kyoto 606-8501, Japan, ${ }^{28}$ Department of Astronomy, Graduate School of Science, Kyoto University, Kitashirakawa Oiwake-cho, Sakyo-ku, Kyoto 6068502, Japan, ${ }^{29}$ Institute of Particle and Nuclear Studies, High Energy Accelerator Research Organization, 1-1 Oho, Tsukuba, Ibaraki 305-0801, Japan, ${ }^{30}$ University of Pisa, Polo Fibonacci, Largo B. Pontecorvo, 3, 56127 Pisa, Italy, ${ }^{31}$ Astroparticle Physics Laboratory, NASA/GSFC, Greenbelt, Maryland 20771, USA, ${ }^{32}$ Department of Electrical and Electronic Systems Engineering, National Institute of Technology, Ibaraki College, 866 Nakane, Hitachinaka, Ibaraki 312-8508, Japan ${ }^{33}$ Department of Astronomy, University of Maryland, College Park, Maryland 20742, USA, ${ }^{34}$ Department of Physical Sciences, College of Science and Engineering, Ritsumeikan University, Shiga 525-8577, Japan, ${ }^{35}$ Faculty of Science and Engineering, Global Center for Science and Engineering, Waseda University, 3-4-1 Okubo, Shinjuku, Tokyo 169-8555, Japan, ${ }^{36}$ Department of Physics and Astronomy, University of Denver, Physics Building, Room 211, 2112 East Wesley Avenue, Denver, Colorado 80208-6900, USA, ${ }^{37}$ Quantum ICT Advanced Development Center, National Institute of Information and Communications Technology, 4-2-1 Nukui-Kitamachi, Koganei, Tokyo 184-8795, Japan, ${ }^{38}$ College of Science and Engineering, Department of Physics and Mathematics, Aoyama Gakuin University, 5-10-1 Fuchinobe, Chuo, Sagamihara, Kanagawa 252-5258, Japan, ${ }^{39}$ College of Industrial Technology, Nihon University, 1-2-1 Izumi, Narashino, Chiba 275-8575, Japan ${ }^{40}$ RIKEN, 2-1 Hirosawa, Wako, Saitama 351-0198, Japan, ${ }^{41}$ Division of Mathematics and Physics, Graduate School of Science, Osaka City University, 3-3-138 Sugimoto, Sumiyoshi, Osaka 558-8585, Japan, ${ }^{42}$ National Institutes for Quantum and Radiation Science and Technology, 4-9-1 Anagawa, Inage, Chiba 263-8555, Japan, ${ }^{43}$ Nagoya University, Furo, Chikusa, Nagoya 464-8601, Japan, ${ }^{44}$ College of Science, Ibaraki University, 2-1-1 Bunkyo, Mito, Ibaraki 310-8512, Japan 